\title{
Health resource utilisation associated with skeletal-related events in European patients with lung cancer: A subgroup analysis from a prospective multinational study
}

\author{
VITO LORUSSO $^{1,2}$, IGNACIO DURAN ${ }^{3,4}$, CRISTINA GARZON-RODRIGUEZ ${ }^{5}$, DIANA LÜFTNER ${ }^{6}$, AMIT BAHL ${ }^{7}$, \\ JOHN ASHCROFT $^{8}$, GUY HECHMATI $^{9}$, RACHEL WEI $^{10}$, EMMA THOMAS $^{11}$ and HERBERT HOEFELER ${ }^{12}$ \\ ${ }^{1}$ Oncology Institute ASL, Lecce; ${ }^{2}$ National Cancer Institute IRCCS Giovanni Paolo II, Bari, Italy; ${ }^{3}$ Centro Integral \\ Oncologica Clara Campal (CIOCC), Madrid; ${ }^{4}$ University Hospital Virgen del Rocio, Seville; ${ }^{5}$ Catalan Institute of Oncology \\ (ICO) - Bellvitge Biomedical Research Institute (IDIBELL), Barcelona, Spain; ${ }^{6}$ Berlin University of Medicine, Berlin, \\ Germany; ${ }^{7}$ University Hospitals Bristol, Bristol; ${ }^{8}$ Pinderfields General Hospital, Wakefield, UK; ${ }^{9}$ Health Economics, \\ Amgen (Europe) GmbH, Zug, Switzerland; ${ }^{10}$ Department of Biostatistics, Amgen, Inc., Thousand Oaks, CA, USA; \\ ${ }^{11}$ Scientific Publications, Amgen (Europe) GmbH, Zug, Switzerland; ${ }^{12}$ Research Center Ruhr, Witten, Germany
}

Received April 16, 2014; Accepted May 21, 2014

DOI: $10.3892 / \mathrm{mco} .2014 .330$

\begin{abstract}
Bone complications or skeletal-related events (SREs), typically defined as radiation to bone, pathological fractures, surgery to bone and spinal cord compression, occur frequently in patients with bone metastases. As the survival of patients with advanced lung cancer improves, preventing SREs is becoming increasingly clinically relevant. The aim of this analysis was to assess the impact of SREs on health resource utilisation (HRU) in European lung cancer patients with bone metastasis. This multinational, observational study included patients who had at least one SRE in the 97 days prior to enrolment, a life expectancy of $\geq 6$ months and an Eastern Cooperative Oncology Group performance status of 0-2. Data on HRU were retrospectively collected for up to 97 days prior to enrolment with a planned prospective follow-up for up to 18-21 months. The HRU measures included the number and length of inpatient hospitalisations and the number of outpatient visits and procedures. The investigators determined whether each HRU was attributable to a SRE. In total, 135 patients with lung cancer, enrolled at centres in Germany, Italy, Spain and the United Kingdom, contributed 214 SREs to this analysis. The median length [quartile $(\mathrm{Q}) 1, \mathrm{Q} 3$ ] of follow-up ranged from $1.5(0.7,3.3)$ to $5.6(2.0,8.2)$ months across the countries. Overall, $41 \%$ of the SREs required an inpatient stay, with a median $(\mathrm{Q} 1, \mathrm{Q} 3)$ duration of $19.0(6.0,28.0)$ days. Spinal cord compression and surgery to
\end{abstract}

Correspondence to: Professor Vito Lorusso, National Cancer Institute IRCCS Giovanni Paolo II, 65 Orazio Flacco Street, I-70124 Bari, Italy

E-mail: vitolorusso@inwind.it; vitolorusso@me.com

Key words: lung cancer, bone metastases, healthcare resource utilisation, skeletal-related events, Europe, disease burden bone were the SRE types most frequently requiring inpatient stays. Radiation to bone was associated with the largest number of outpatient visits and procedures. All the SREs resulting from bone metastases in patients with lung cancer contribute considerably to HRU and efforts to minimise the incidence of bone complications in these patients through appropriate treatments may help reduce this burden.

\section{Introduction}

In 2012, >400,000 patients in Europe were diagnosed with lung cancer (1). Historically, it has been reported that approximately one-third of these individuals will develop bone metastases $(2,3)$. However, recent advances in treatment, such as the use of inhibitors of the epidermal growth factor receptor and anaplastic lymphoma kinase oncogenes (4), have extended the life expectancy of patients with lung cancer, suggesting that bone metastases and their associated complications have become more clinically relevant (5).

Metastatic bone complications, otherwise known as skeletal-related events (SREs), are common in patients with lung cancer; a large, randomised, placebo-controlled, phase 3 study demonstrated that the rate of SREs in patients with lung cancer and other solid tumours (excluding breast and prostate cancer) was $46 \%$ over a 21 -month period (6). SREs are typically defined as radiation to bone, pathological fractures, surgery to bone and spinal cord compression. SREs may cause debilitating pain and reduced mobility, resulting in a reduction in the quality of life and significant decreases in physical well-being and functional independence $(7,8)$. Radiation to bone, often used to treat pain, may itself cause transient increases in pain ('pain flare') (9), while opioids, also commonly used to alleviate pain, are associated with severe side effects, including nausea and constipation (10), as well as psychological association and fears (11). In addition to causing persistent pain, pathological fractures reduce load-bearing capacity and restrict movement. 
Furthermore, spinal cord compression is a serious complication, which may even lead to paralysis $(2,12)$. Surgery to bone often requires lengthy inpatient stays, which may be traumatic for patients and their families (13). Thus, SREs impose a substantial burden on patients.

In addition to the clinical and patient impact, SREs place a considerable burden onto healthcare systems. Several retrospective studies have highlighted the increase in treatment costs as patients with lung cancer progress to metastatic bone disease and experience associated SREs (14-18). A prospective study in France also highlighted the high cost of care of patients with SREs, with $50 \%$ of the cost for the treatment of patients with lung cancer who have bone metastases being linked to the occurrence of SREs (19). Although these data provide some insight into the impact that lung cancer-associated SREs have on healthcare systems, there is generally a lack of prospective data from a broader European population. Increasing awareness of the burden of these bone complications will highlight the need for appropriate patient management and aid healthcare planning through a better understanding of the resources commonly required in treating SREs.

A prospective, observational, multinational study was conducted to evaluate the health resource utilisation (HRU) associated with SREs in patients with bone metastases secondary to cancer of the prostate, breast or lung and multiple myeloma in Canada, Germany, Italy, Spain, the United Kingdom (UK) and the United States of America. Data on the HRU associated with all tumour types in Europe and the financial cost of SREs associated with solid tumours in Europe have previously been reported $(20,21)$. In the present study, we assessed the data for the subset of patients with lung cancer from Germany, Italy, Spain and the UK, since the European approach to patient management is fairly congruent.

\section{Materials and methods}

Patients. Eligible patients were aged $\geq 18$ years, with bone metastases secondary to lung cancer and a life expectancy of $\geq 6$ months (as judged by the treating physician). Patients were required to have an Eastern Cooperative Oncology Group (ECOG) performance status of 0,1 or 2 and to have experienced $\geq 1$ SRE in the 97 days prior to enrolment (index SRE). For patients who had experienced $>1$ SRE in the 97 days prior to enrolment, the index SRE was classified based on the following hierarchy: spinal cord compression, surgery to bone, pathological fracture and radiation to bone. Patients who were already enrolled into an investigational drug trial for the treatment of bone metastases or prevention of SREs were excluded from this study.

Study design. Patient demographics and medical history (including time since bone metastasis detection) were collected at enrolment. Data were collected both retrospectively and prospectively: HRU data for each patient were collected retrospectively through chart review for all SREs occurring in the 97-day period prior to enrolment and prospectively for the duration of each participant's involvement in the study. Treating physicians independently attributed HRU to SREs. If radiation or surgery to bone was performed as a result of another SRE (i.e., to treat a primary SRE), the physicians had the option of attributing the HRU to the primary SRE. However, in these cases, the inclusion of radiation or surgery to bone as discrete SREs with no associated HRU in the analysis would result in an underestimation of the mean HRU for these two types of SREs. Thus, in such cases, these SREs were excluded from the analysis. Physicians were required to report all SRE-related HRU data at least every 90 days during the prospective data collection period.

The planned follow-up period was 18-21 months. The HRU outcome measures recorded were as follows: number, duration and facility type of inpatient stays; number and facility type of outpatient visits; number and specific type of procedures (e.g., imaging, radiation therapy or outpatient procedures, such as surgery); number of emergency department visits; number of home health visits; and previous bisphosphonate use and duration of use.

Informed consent was required prior to the collection of patient data. The study was performed in compliance with the Helsinki Declaration.

Statistical analyses. HRU was summarised by SRE type; the details have been previously described (20). The mean HRU per SRE type was calculated by dividing the total HRU attributed to a specific SRE type by the total number of SREs of the same type. The mean duration of inpatient stay per SRE was calculated as the total number of inpatient days divided by the total number of SREs that were associated with at least one inpatient stay (if a SRE contributed multiple inpatient stays, the total duration of all these inpatient stays was used).

The data are primarily reported as both mean and median values, where appropriate. The mean values describe the total resources used at a population level: information that is required for healthcare policy decisions (20). The median values are also reported to illustrate the distribution of data when sample sizes are small and to describe the typical HRU for an individual patient.

\section{Results}

Study cohort. A total of 135 patients with a primary diagnosis of lung cancer were enrolled (Table I). Of the 135 patients, 34 were from Germany, 43 from Italy, 41 from Spain and 17 from the UK. Baseline characteristics were generally similar across the four countries. The mean age \pm standard deviation (SD) ranged from $60.3 \pm 10.3$ to $66.6 \pm 11.4$ years across all countries. Data on the number of patients who had received bisphosphonates at any point prior to enrolment revealed that, compared with patients from the other countries, patients in the UK were less likely to have received bisphosphonate treatment. This may be associated with the fact that fewer patients in the UK had previously experienced SREs prior to the 97-day period preceding the signing of informed consent. The median time to enrolment since primary cancer diagnosis ranged from 2.2 to 3.5 months across all countries and was similar to the median time since the detection of bone metastasis (range, 1.7-2.5 months). This suggests that, in a large proportion of patients, diagnoses were made at a late stage of the disease, with patients likely to have metastases at the time of diagnosis. The median length of the follow-up was 1.5-5.6 months across the four countries. 
Table I. Baseline demographics and disease history.

\begin{tabular}{|c|c|c|c|c|}
\hline & Germany $(n=34)$ & Italy $(n=43)$ & Spain $(n=41)$ & $\mathrm{UK}(\mathrm{n}=17)$ \\
\hline \multicolumn{5}{|l|}{ Follow-up time, months } \\
\hline Mean (SD) & $5.5(4.1)$ & $5.6(4.2)$ & $3.7(3.8)$ & $3.2(4.2)$ \\
\hline Median & 5.6 & 4.8 & 2.6 & 1.5 \\
\hline$(\mathrm{Q} 1, \mathrm{Q} 3)$ & $(2.0,8.2)$ & $(1.9,8.0)$ & $(1.2,4.4)$ & $(0.7,3.3)$ \\
\hline Male, n (\%) & $24(70.6)$ & $26(60.5)$ & $28(68.3)$ & $10(58.8)$ \\
\hline \multicolumn{5}{|l|}{ Ethnic group, n (\%) } \\
\hline White or Caucasian & $33(97.1)$ & $43(100.0)$ & $40(97.6)$ & $17(100.0)$ \\
\hline Other & $1(2.9)$ & $0(0.0)$ & $1(2.4)$ & $0(0.0)$ \\
\hline \multicolumn{5}{|l|}{ Age, years } \\
\hline Mean (SD) & $63.0(10.6)$ & $64.1(9.1)$ & $60.3(10.3)$ & $66.6(11.4)$ \\
\hline$\geq 65, \mathrm{n}(\%)$ & $15(44.1)$ & $24(55.8)$ & $15(36.6)$ & $10(58.8)$ \\
\hline \multicolumn{5}{|l|}{ ECOG performance status, n (\%) } \\
\hline 0 & $4(11.8)$ & $12(27.9)$ & $7(17.0)$ & $0(0.0)$ \\
\hline 1 & $18(52.9)$ & $17(39.5)$ & $17(41.5)$ & $5(29.4)$ \\
\hline 2 & $12(35.3)$ & $14(32.6)$ & $17(41.5)$ & $12(70.6)$ \\
\hline History of SREs ${ }^{\mathrm{a}}, \mathrm{n}(\%)$ & $22(64.7)$ & $24(55.8)$ & $27(65.9)$ & $2(11.8)$ \\
\hline \multicolumn{5}{|l|}{$\begin{array}{l}\text { Time to enrolment since primary } \\
\text { cancer diagnosis, months }\end{array}$} \\
\hline Mean (SD) & $7.8(15.0)$ & $7.0(9.5)$ & $8.1(10.8)$ & $14.2(32.3)$ \\
\hline Median & 3.2 & 2.6 & 3.5 & 2.2 \\
\hline (Q1, Q3) & $(1.2,6.5)$ & $(1.4,8.5)$ & $(1.5,10.4)$ & $(1.2,5.2)$ \\
\hline \multicolumn{5}{|l|}{$\begin{array}{l}\text { Time since bone metastasis } \\
\text { detection, months }\end{array}$} \\
\hline Mean (SD) & $4.5(12.9)$ & $4.2(4.2)$ & $4.6(7.3)$ & $2.6(2.3)$ \\
\hline Median & 1.9 & 2.5 & 1.8 & 1.7 \\
\hline (Q1, Q3) & $(0.85,3.4)$ & $(1.4,6.7)$ & $(1.2,4.4)$ & $(1.2,3.2)$ \\
\hline Previous bisphosphonate use ${ }^{\mathrm{b}}, \mathrm{n}(\%)$ & $22(64.7)$ & $21(48.8)$ & $16(39.0)$ & $5(29.4)$ \\
\hline \multicolumn{5}{|l|}{$\begin{array}{l}\text { Duration of previous bisphosphonate } \\
\text { use }^{b}, \text { months }\end{array}$} \\
\hline Mean (SD) & $5.0(16.1)$ & $2.7(3.7)$ & $3.3(7.8)$ & $1.0(0.8)$ \\
\hline Median & 1.2 & 1.7 & 0.9 & 0.9 \\
\hline (Q1, Q3) & $(0.4,2.0)$ & $(0.2,3.1)$ & $(0.4,1.7)$ & $(0.6,1.1)$ \\
\hline
\end{tabular}

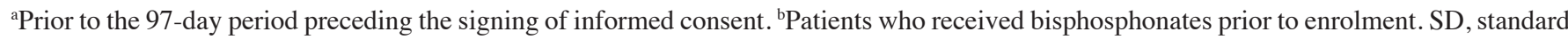
deviation; Q1, first quartile; Q3, third quartile; ECOG, Eastern Cooperative Oncology Group; SRE, skeletal-related event.

Skeletal-related events. Eligible patients experienced a total of 239 SREs. Analysis of the crude SRE data (including SREs that were excluded from the HRU analysis) found the number of SREs per patient-year varied by country (2.8 for Germany, 2.1 for Italy, 3.2 for Spain and 3.9 for the UK). Following removal of the SREs for which HRU was attributed to another (primary) SRE, 214 SREs were included in the study (Fig. 1). Radiation to bone was the most common SRE (140 events); pathological fractures, surgery to bone and spinal cord compression were less common (38, 21 and 15 events, respectively).

\section{Health resource utilisation}

Inpatient stays. Overall, 4 in every 10 SREs (87 of 214 SREs) required an inpatient stay (Fig. 2). Inpatient stays were slightly less frequent in the UK, being required in approximately 3 in every 10 SREs. Spinal cord compression and surgery to bone were the SREs most likely to result in an inpatient stay (80.0 and $71.4 \%$ of SREs, respectively). In Germany, hospitalisation for radiation to bone was also relatively common, with 1 in 2 SREs requiring an inpatient stay (50.0\%). In Spain and Italy, patients were more likely to be hospitalised for pathological fractures compared with the other countries (60.0 and $58.3 \%$, respectively, compared with the overall mean of $44.7 \%$ for all the countries), although the data should be interpreted with caution due to the small number of pathological fracture events in total.

Data on the duration of inpatient stays were collected for 86 of the 87 SREs that required hospitalisation. The median [quartile (Q)1, Q3] length of stay per SRE that resulted in hospitalisation was 19.0 (6.0, 28.0) days (Fig. 3). Across all 


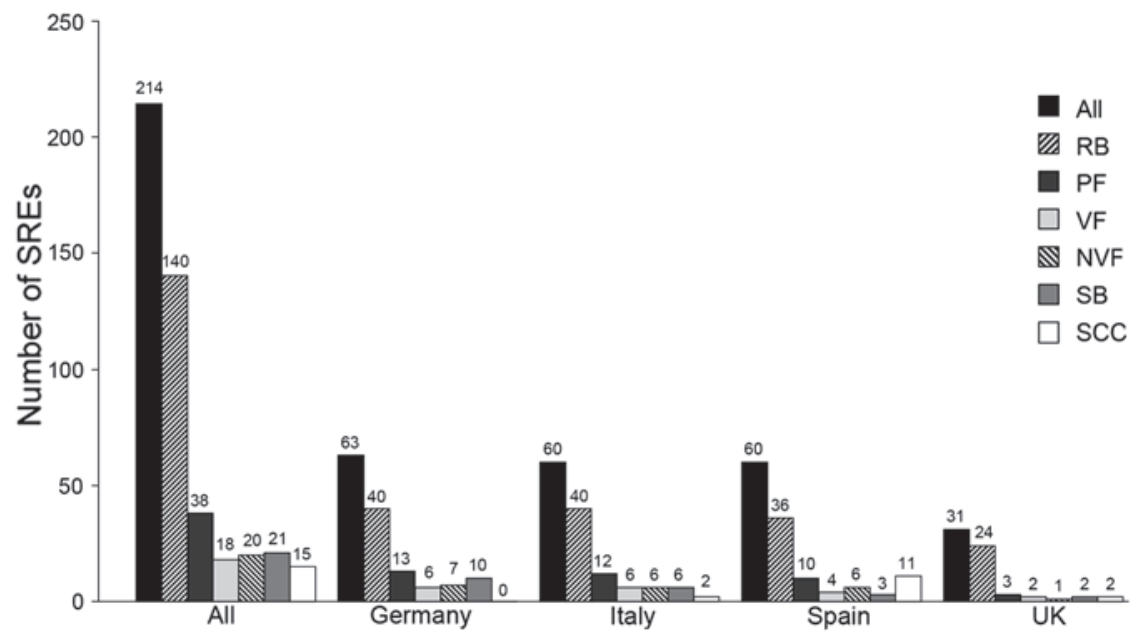

Figure 1. Number of SREs included in the health resource utilisation analysis by country and by SRE type. VF and NVF are subsets of PF. SRE, skeletal-related event; RB, radiation to the bone; $\mathrm{PF}$, pathological fracture; $\mathrm{VF}$, vertebral fracture; $\mathrm{NVF}$, non-vertebral fracture; SB, surgery to the bone; SCC, spinal cord compression.

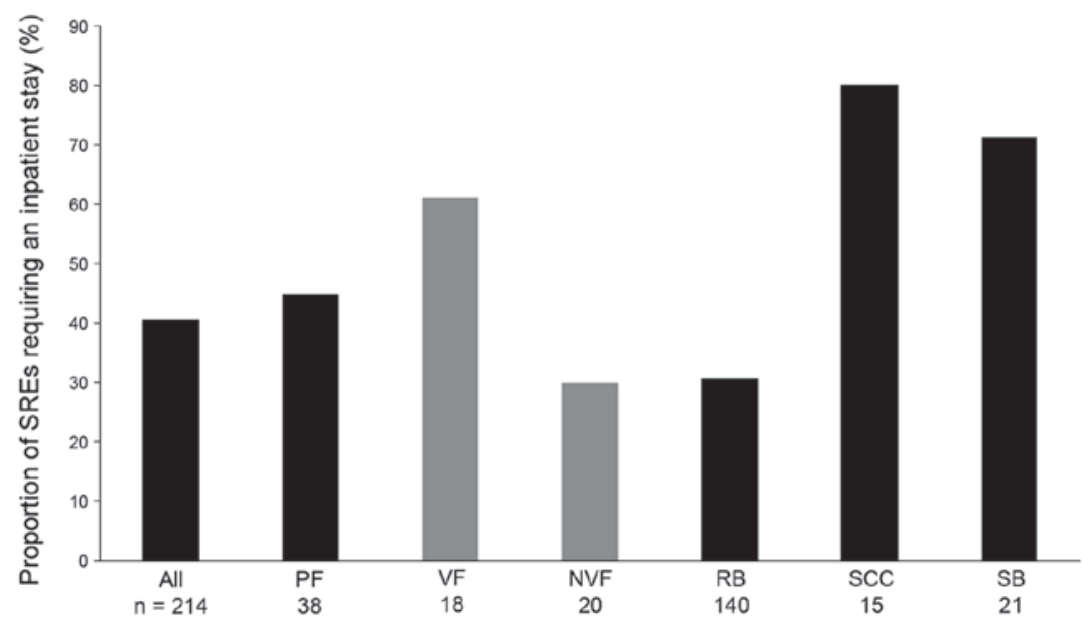

Figure 2. Proportion of SREs requiring an inpatient stay. VF and NVF are subsets of PF. SRE, skeletal-related event; n, number of SREs; PF, pathological fracture; VF, vertebral fracture; NVF, non-vertebral fracture; RB, radiation to the bone; SCC, spinal cord compression; SB, surgery to the bone.

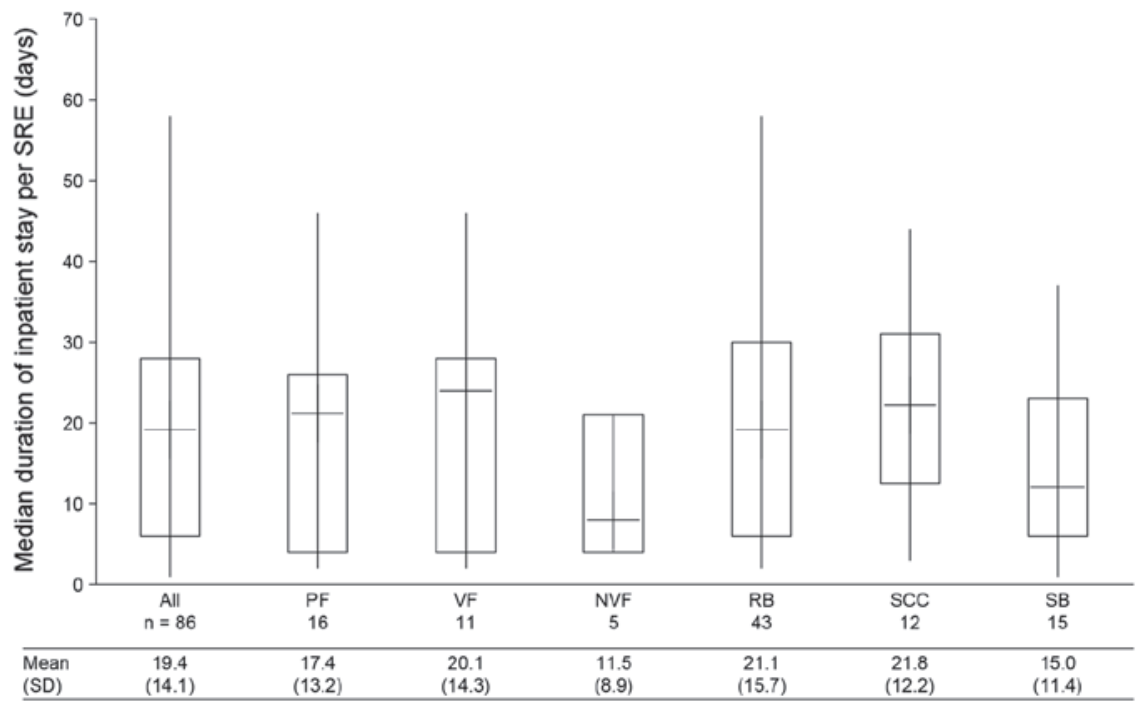

Figure 3. Duration of inpatient stay per SRE. Data in the box and whisker plot are shown as median (horizontal line within box) + interquartile range (box) + range (whiskers) and include only SREs requiring an inpatient stay. If a SRE resulted in multiple inpatient stays, the total duration of all the inpatient stays was used. VF and NVF are subsets of PF. SRE, skeletal-related event; SD, standard deviation; n, number of SREs; PF, pathological fracture; VF, vertebral fracture; NVF, non-vertebral fracture; RB, radiation to the bone; SCC, spinal cord compression; SB, surgery to the bone. 


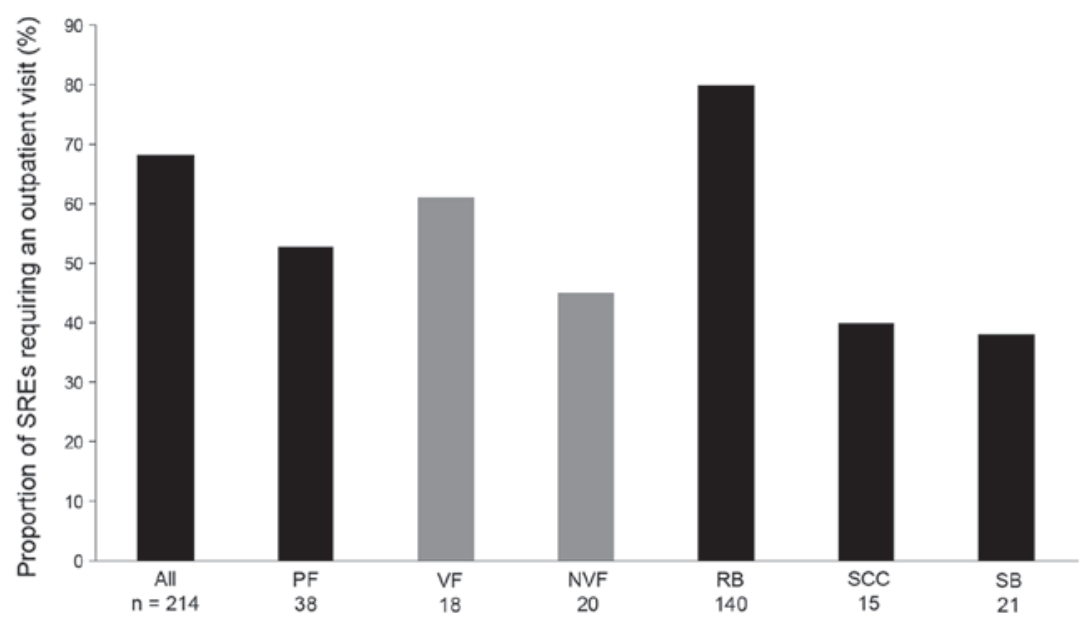

Figure 4. Proportion of SREs requiring an outpatient visit. VF and NVF are subsets of PF. SRE, skeletal-related event; $\mathrm{n}$, number of SREs; PF, pathological fracture; VF, vertebral fracture; NVF, non-vertebral fracture; RB, radiation to the bone; SCC, spinal cord compression; SB, surgery to the bone.

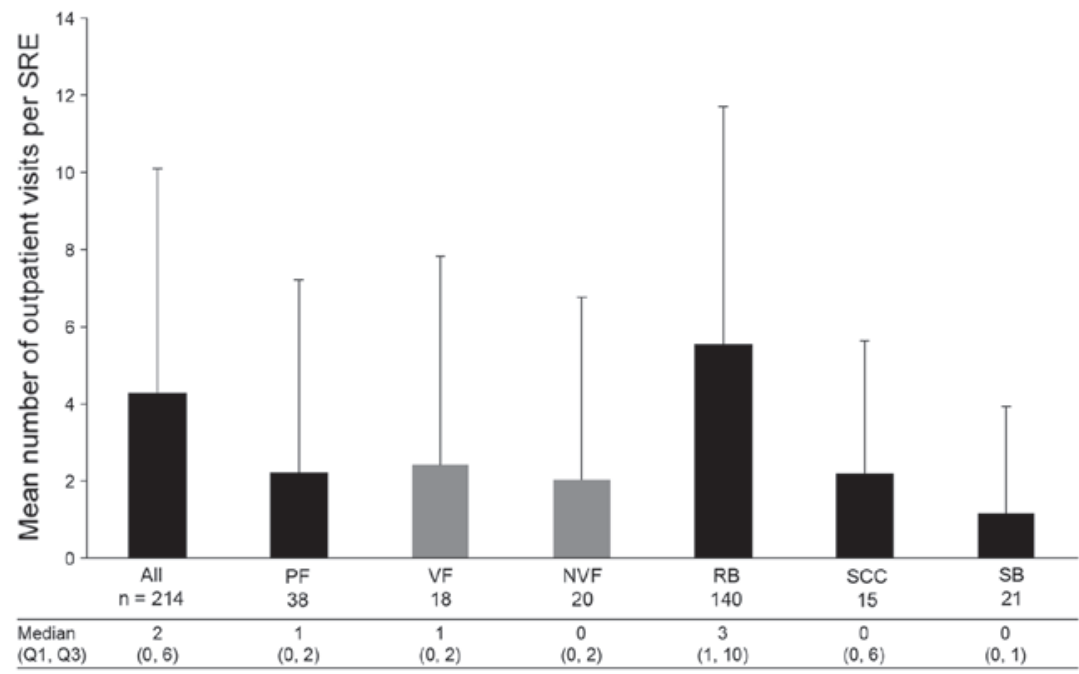

Figure 5. Number of outpatient visits per SRE. Data in the bar chart are shown as mean + standard deviation. VF and NVF are subsets of PF. SRE, skeletal-related event; Q1, first quartile; Q3, third quartile; n, number of SREs; PF, pathological fracture; VF, vertebral fracture; NVF, non-vertebral fracture; RB, radiation to the bone; SCC, spinal cord compression; SB, surgery to the bone.

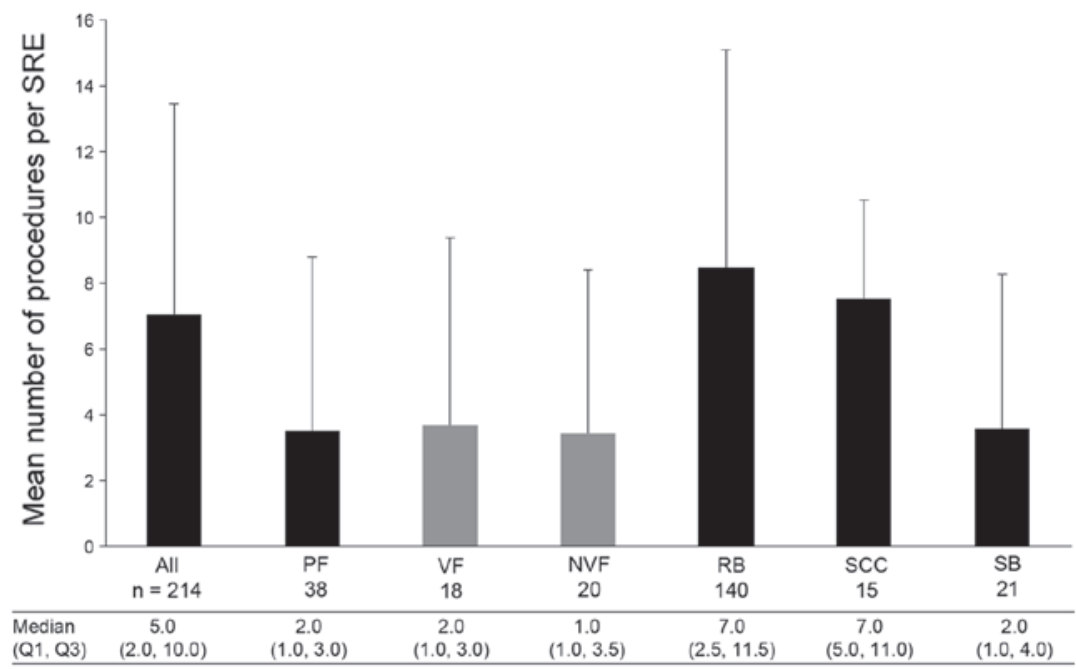

Figure 6. Number of procedures performed per SRE. Data are presented as mean + standard deviation. VF and NVF are subsets of PF. SRE, skeletal-related event; Q1, first quartile; Q3, third quartile; n, number of SREs; PF, pathological fracture; VF, vertebral fracture; NVF, non-vertebral fracture; RB, radiation to the bone; SCC, spinal cord compression; SB, surgery to the bone. 
countries, spinal cord compression and pathological fracture had the longest median length of stays $[22.0(12.5,31.0)$ and $21.0(4.0,26.0)$ days, respectively] and surgery to bone the shortest $[12.0(6.0,23.0)$ days]. Patients were most likely to be treated in oncology units; general, radiation and surgical units were also frequently used (data not shown).

Outpatient visits. Across the four countries, two-thirds (mean $68.2 \%$ ) of SREs required an outpatient visit (Fig. 4). The rates were higher in Italy and the UK (80.0 and $83.9 \%$ of events, respectively) compared with those in Germany or Spain (58.7 and 58.3\%, respectively). Radiation to bone most frequently required outpatient visits ( $80.0 \%$ of events), while surgery to bone required the fewest visits (38.1\% of events). This pattern was consistent across the countries, with the exception of Germany, where pathological fractures were least likely to require a visit $(23.1 \%)$ and was low vs. the mean of the overall study population (52.6\%).

The mean number $( \pm$ SD) of outpatient visits required per SRE was $4.3( \pm 5.8)$, with radiation to bone requiring the most $[5.6( \pm 6.1)]$ and surgery to bone the fewest $[1.2( \pm 2.8)]$ visits (Fig. 5). Patients in Germany had the highest mean number of outpatient visits per SRE $[6.3( \pm 8.1)]$ and patients in the UK had the lowest $[2.9( \pm 3.0)]$. Patients in the UK had far fewer visits per radiation to bone event [mean, $2.6( \pm 2.6)]$ compared with those in the other three countries [ranging between $4.5( \pm 4.4)$ and $8.6( \pm 8.1)]$.

Procedures. Almost all of the SREs required at least one procedure (96.7\%). Across all the SRE types and countries, a mean $( \pm \mathrm{SD})$ of $7.1( \pm 6.4)$ procedures were required per SRE (Fig. 6). The most common type of procedure was external beam radiation, with a mean $( \pm \mathrm{SD})$ of $4.1( \pm 6.0)$ procedures per SRE, followed by intensity-modulated radiotherapy [1.6 $( \pm 4.4)]$ and computed tomography $[0.3( \pm 0.5)]$. In all countries apart from the UK, radiation to bone required the highest number of procedures per SRE. In Germany, a mean of $13.9( \pm 7.1)$ procedures were required per radiation to bone event; in the UK, only $3.4( \pm 2.4)$ procedures were performed per radiation to bone event. The majority of the procedures were performed in the outpatient setting [4.4 $( \pm 5.8)$ per SRE], with radiation to bone being the main contributor to this mean value $[5.7( \pm 6.1)$ procedures in the outpatient setting per SRE]. The procedures were a major contributor to SRE-related inpatient stays, with a mean $( \pm \mathrm{SD})$ of $2.5( \pm 4.4)$ procedures requiring hospitalisation per SRE, with spinal cord compression was the most significant contributor $(5.1 \pm 3.7$ procedures resulting in an overnight stay per SRE).

Emergency department and home health visits. Emergency department visits and home health visits were less frequently reported compared to other measures of HRU (3.3 and $0.9 \%$ of SREs, respectively).

\section{Discussion}

To the best of our knowledge, this is the first combined retrospective and prospective study to investigate HRU associated with SREs in patients with advanced lung cancer in Europe. Furthermore, this is the first study in which HRU was assigned to specific SREs independently by investigators. Therefore, HRU was recorded only when the treating physician considered it to be a direct result of a SRE, as opposed to the underlying disease. In line with other previous studies $(22,23)$, all the SREs resulting from bone metastases secondary to lung cancer were associated with substantial HRU.

Inpatient stays were common (40.7\% of SREs), with hospitalisations lasting a median of $\sim 3$ weeks. The proportion of patients with lung cancer requiring an inpatient stay was significantly higher compared to that of patients with breast or prostate cancer $(23,24)$. The patients were also more likely to have a worse performance status, which may contribute to an increased need for hospitalisation on experiencing a SRE. In addition, outpatient visits were necessary for the majority of SREs (68.2\%), with several patients requiring multiple visits. Similarly, almost every SRE required a procedure and multiple procedures were common.

Although spinal cord compression and surgery to the bone reportedly occur less frequently compared with the other SREs (6), the results of this analysis demonstrated that they were more likely to be associated with inpatient stays; spinal cord compression was associated with the longest stays among all SREs (median of 22.0 days). Conversely, although radiation to bone was the SRE least likely to be associated with an inpatient stay, this SRE occurs frequently (6). Therefore, the associated HRU is also likely to be substantial. Inpatient stays required for radiation to bone were also lengthy, indicating that radiation to bone may impose a significant burden on healthcare resources. Radiation to bone also required the highest number of outpatient visits and procedures per SRE, suggesting it is a major contributor to HRU.

The patterns of HRU were generally similar across countries, although certain exceptions were noted. In the UK, radiation to bone was less likely to require multiple outpatient visits and procedures compared to the other countries, possibly reflecting a preference for single- as opposed to multiple-fraction radiotherapy $(25,26)$. Overall, the proportion of SREs requiring an outpatient visit was highest in the UK and Italy. Patients in these countries were also less likely to be hospitalised, suggesting a preference for outpatient management of SREs.

In our study, the incidence of previous treatment with bisphosphonates for the prevention of SREs was low, particularly in the UK. This reflects the lower percentage of patients in the UK who had a history of SREs compared with the other countries. In addition, the length of time over which patients had been treated with bisphosphonates was short. The general lack of treatment observed in all the countries may have resulted in a high SRE incidence, contributing to the high levels of HRU required by these patients.

Undertreatment with bone-targeted agents in patients with bone metastases has been observed across a number of solid tumour types (27) and appears to be a particular problem in lung cancer. A study focusing on patients with lung cancer reported that as few as $38 \%$ of the patients received the bisphosphonate zoledronic acid, which was the most commonly used bone-targeted agent at the time (28). A prospective chart audit investigating bisphosphonate use in individuals with lung cancer revealed that $44 \%$ of the patients were not receiving treatment at the time of the study (29). These trends may be due to the perceived short life expectancy of patients with lung cancer (29) and may also reflect the concern of adding nephrotoxic bone-targeted agents to the nephrotoxic platinum-based 
chemotherapy that is commonly used in the treatment of lung cancer. However, SREs have been shown to commonly occur within the first 2 months of diagnosis (15), with individuals experiencing events every 3-6 months thereafter (2). Given that the median survival of patients with advanced lung cancer is currently $\sim 12$ months (5), a short life expectancy should no longer constitute a reason for not prescribing treatment with bone-targeted agents. Furthermore, avoiding SREs and their associated complications, including increased requirements for medical care and lengthy hospitalisations, may be of particular concern to patients with a limited expected lifespan.

The timing of the initiation of treatment with bone-targeted agents in patients with lung cancer may also be an area of concern, considering that these patients are often diagnosed at an advanced stage. The similar values reported in this study for time since primary cancer diagnosis and time since bone metastasis detection suggest that several patients presented with metastatic bone disease at the time of primary cancer diagnosis. This may also suggest that, up to that point, the patients had not received treatment with bone-targeted agents and may already have been experiencing pain associated with bone complications.

There were a number of limitations associated with this study, as previously reported by Hoefeler et al (20). The key considerations included the limited duration of the follow-up. In addition, the numbers of patients enrolled were also limited, leading to small sample sizes in the majority of the SRE subsets, i.e., spinal cord compression, surgery to bone and pathological fractures. It should be noted that the relative proportions of SREs reported in this study are not representative of the real-world distribution of SRE types, as they are affected by the index SRE recruitment target (i.e., prespecified numbers of each SRE type) and inclusion criteria. Additionally, although pain was not defined as an SRE, it is a common problem in patients with poorly treated bone metastases and may contribute to additional HRU. In general, these limitations may be expected to result in the underestimation of the overall HRU associated with SREs and thus suggest that our data are conservative.

This study highlights the considerable resource requirements imposed on healthcare services by SREs in patients with lung cancer and bone metastases. In addition to lowering the burden on patients, preventing SREs in patients with bone metastases secondary to lung cancer through better access to effective and appropriate treatment may substantially reduce HRU.

\section{Acknowledgements}

The authors would like to thank Dr Kim Allcott (PhD) of Oxford PharmaGenesis ${ }^{\mathrm{TM}}$ Ltd., (Oxford, UK), who provided editorial support. Funding for this support was provided by Amgen (Europe) $\mathrm{GmbH}$. Additional statistical analysis and support was provided by Prayashi Ghelani, who is an employee of Amgen. Ignacio Duran has acted as a member of advisory boards for Amgen. Cristina Garzon-Rodriguez has acted as a member of advisory boards for Amgen. Diana Lüftner has received honoraria for presentations and has acted as a member of advisory boards for Amgen. Amit Bahl has acted as a member of advisory boards for Amgen and has received honoraria for presentations. John Ashcroft has acted as a member of advisory boards for Amgen and Novartis. Guy Hechmati is an employee of Amgen and holds stock. Rachel Wei is an employee of Amgen and holds stock. Emma Thomas is an employee of Amgen and holds stock. Herbert Hoefeler has acted as a member of advisory boards for Amgen. This study was funded by Amgen.

\section{References}

1. Steliarova-Foucher E, O'Callaghan M, Ferlay J, et al: European Cancer Observatory: Cancer Incidence, Mortality, Prevalence and Survival in Europe. Version 1.0 (September 2012). European Network of Cancer Registries, International Agency for Research on Cancer. http://eco.iarc.fr. Accessed June 18, 2013.

2. Coleman RE: Clinical features of metastatic bone disease and risk of skeletal morbidity. Clin Cancer Res 12: 6243s-6249s, 2006

3. Galasko C: The anatomy and pathways of skeletal metastases. In: Bone Metastases. Weiss L and Gilbert A (eds). GK Hall, Boston, pp49-63, 1981.

4. Mok TS: Personalized medicine in lung cancer: what we need to know. Nat Rev Clin Oncol 8: 661-668, 2011.

5. Brodowicz T, O'Byrne $\mathrm{K}$ and Manegold C: Bone matters in lung cancer. Ann Oncol 23: 2215-2222, 2012.

6. Rosen LS, Gordon D, Tchekmedyian NS, et al: Long-term efficacy and safety of zoledronic acid in the treatment of skeletal metastases in patients with nonsmall cell lung carcinoma and other solid tumors: a randomized, phase III, double-blind, placebo-controlled trial. Cancer 100: 2613-2621, 2004.

7. Costa L, Badia X, Chow E, Lipton A and Wardley A: Impact of skeletal complications on patients' quality of life, mobility, and functional independence. Support Care Cancer 16: 879-889, 2008.

8. Mercadante S: Malignant bone pain: pathophysiology and treatment. Pain 69: 1-18, 1997.

9. Loblaw DA, Wu JS, Kirkbride P, et al: Pain flare in patients with bone metastases after palliative radiotherapy - a nested randomized control trial. Support Care Cancer 15: 451-455, 2007.

10. Coleman RE: Skeletal complications of malignancy. Cancer 80 (Suppl 8): 1588-1594, 1997.

11. National Collaborating Centre for Cancer (UK): Opioids in palliative care: safe and effective prescribing of strong opioids for pain in palliative care of adults. Full guideline, May, 2012

12. Loblaw DA, Perry J, Chambers A and Laperriere NJ: Systematic review of the diagnosis and management of malignant extradural spinal cord compression: the Cancer Care Ontario Practice Guidelines Initiative's Neuro-Oncology Disease Site Group. J Clin Oncol 23: 2028-2037, 2005.

13. Katzer A, Meenen NM, Grabbe F and Rueger JM: Surgery of skeletal metastases. Arch Orthop Trauma Surg 122: 251-258, 2002.

14. Body JJ, Chevalier P, Gunther O, Hechmati G and Lamotte M: The economic burden associated with skeletal-related events in patients with bone metastases secondary to solid tumors in Belgium. J Med Econ 16: 539-546, 2013.

15. Delea T, Langer C, McKiernan J, et al: The cost of treatment of skeletal-related events in patients with bone metastases from lung cancer. Oncology 67: 390-396, 2004.

16. Delea T, McKiernan J, Brandman J, et al: Retrospective study of the effect of skeletal complications on total medical care costs in patients with bone metastases of breast cancer seen in typical clinical practice. J Support Oncol 4: 341-347, 2006.

17. Pockett RD, Castellano D, McEwan P, Oglesby A, Barber BL and Chung K: The hospital burden of disease associated with bone metastases and skeletal-related events in patients with breast cancer, lung cancer, or prostate cancer in Spain. Eur J Cancer Care (Engl) 19: 755-760, 2010.

18. Schulman KL and Kohles J: Economic burden of metastatic bone disease in the U.S. Cancer 109: 2334-2342, 2007.

19. Decroisette C, Monnet I, Berard H, et al: Epidemiology and treatment costs of bone metastases from lung cancer: a French prospective, observational, multicenter study (GFPC 0601). J Thorac Oncol 6: 576-582, 2011.

20. Hoefeler H, Duran I, Hechmati G, et al: Health resource utilization associated with skeletal-related events in patients with bone metastases: results from a multinational retrospective - prospective observational study - a cohort from 4 European countries. J Bone Oncol (In press). 
21. Hechmati G, Cure S, Gouepo A, et al: Cost of skeletal-related events in European patients with solid tumours and bone metastases: data from a prospective multinational observational study. J Med Econ 16: 691-700, 2013.

22. Lüftner D, Lorusso V, Duran I, et al: Health resource utilization associated with skeletal-related events in patients with advanced breast cancer: results from a prospective, multinational observational study. Submitted for publication, 2013.

23. Hoefeler H, Duran I, Hechmati G, et al: Health resource utilization (HRU) associated with skeletal-related events (SREs) by tumour type in patients with bone metastases/lesions: European analysis of a prospective multinational observational study. Eur J Cancer 47: S262-S263, 2011.

24. Lüftner D, Lorusso V, Duran I, et al: Health resource utilization (HRU) associated with skeletal-related events (SREs) in advanced breast cancer patients with bone metastases: results from a prospective multinational observational study. Cancer Res 71 (24 Suppl): abstract P4-16-09, 2011.

25. Royal College of Radiologists: Single fraction radiotherapy for bone metastases. http://www.rcr.ac.uk/docs/oncology/pdf/ bonemetsreport.pdf. Accessed June 27, 2013.
26. Williams MV, Summers ET, Drinkwater K and Barrett A: Radiotherapy dose fractionation, access and waiting times in the countries of the UK in 2005. Clin Oncol (R Coll Radiol) 19: 273-286, 2007.

27. Casas A, Lebret T, Cavo M, et al: Insights into the management of bone metastases: a comprehensive European survey. Support Care Cancer 20: S88, 2012.

28. Calderone R, Nimako K, Leary A, Popat S and O'Brien ME: Under usage of zoledronic acid in non-small cell lung cancer patients with metastatic bone disease - a short communication. Eur J Cancer 47: 1603-1605, 2011.

29. Woll PJ, Flinois A, Schoen P, Shepherd S and Haynes I: Insights into the management of bone metastases in patients with lung cancer: A comprehensive European survey. Eur J Cancer 49: abstract 3473, 2013. 